\title{
Fluxo de vapor de água atmosférico na obtenção do resíduo ET-P em três macrorregiões brasileiras
}

\author{
Enilson P. Cavalcanti ${ }^{1}$, Manoel F. Gomes Filho ${ }^{1} \&$ Wagner de A. Bezerra ${ }^{1}$
}

\begin{abstract}
RESU M O
São analisados, neste trabalho, a precipitação, a evapotranspiração e o resíduo (ET-P) para três áreas de controle de 10 por 10 graus de latitude e longitude sobre as regiões Nordeste, Norte e Sudeste do Brasil. 0 fluxo de vapor d'água resultante é calculado e comparado com o resíduo. As análises são feitas a partir dos dados de reanálise mensais do National Center for Atmospheric Research/National Centers for Environmental Prediction NCAR/N CEP, para o período de 1958 a 1998. Aspectos da variação sazonal e interanual dos elementos citados são apresentados para cada uma das áreas e, entre os resultados obtidos, destacam-se a boa correlação entre 0 resíduo e o fluxo de vapor d'água resultante, com coeficientes de determinação de 0,86, 0,84 e 0,74 para as áreas N ordeste, Norte e Sudeste, respectivamente.
\end{abstract}

Palavras-chave: clima, precipitação, evapotranspiração

\section{Atmospheric water vapor flux in the determination of the residual ET-P in three Brazilian macro regions}

\begin{abstract}
This paper presents an analysis of precipitation, evapotranspiration and ET-P residuals for three $10^{\circ}$ by $10^{\circ}$ latitude/longitude control areas over the northeast, north and southeast of Brazil. The resulting water vapor flux is calculated and compared to the residual. The analysis is performed through the NCAR/NCEP reanalysis data for the period from 1958 to 1998 . Some aspects of both seasonal and interannual variation of these variables are presented for each depicted area. The main result is the good correlation between the residuals and the resulting water vapor flux with determination coefficients of $0.86,0.84$ and 0.74 for these respective regions.
\end{abstract}

Key words: climate, precipitation, evapotranspiration 


\section{INTRODUÇÃO}

Acreditava-se, outrora, que a precipitação pluvial era originada principalmente pela evaporação local (Brubaker et al., 1993). Desse ponto de vista, se conclui que um simples aumento da evapotranspiração ou evaporação local aumentaria também a precipitação pluvial mas, no final da década de 1930, se iniciaram as medições de elementos meteorológicos nas camadas superiores da atmosfera possibilitando estudos sobre o armazenamento de vapor d'água e seu transporte anulando, assim, este paradigma (Benton \& Stoque, 1954; Peixoto, 1959; Hastenrath, 1966; Rasmusson, 1967; Rasmusson, 1971; Howarth, 1983).

O vapor d'água é armazenado na atmosfera e transportado pela circulação geral. Segundo Howarth (1983) considerando-se que a água precipitável média global é de $25 \mathrm{~mm}$ e que a precipitação média global é de $1.000 \mathrm{~mm}$ ano-1, verifica-se que o tempo de permanência da água na atmosfera é de 9 a 10 dias. Se o calor latente de condensação for tomado como $2,5 \times 10^{6} \mathrm{~J} \mathrm{~kg}^{-1}$, a quantidade de energia liberada na atmosfera será de aproximadamente $1,28 \times 10^{24} \mathrm{~J}_{\text {ano }}{ }^{-1}$, equivalente a $35 \%$ da energia solar absorvida pelo sistema terraatmosfera, no mesmo período, o que exemplifica bem a importância do vapor d'água nos processos físicos e dinâmicos da atmosfera.

Limitações impostas por falhas freqüentes nas séries temporais de radiossondagens (dias sem sondagens) e sua má distribuição espacial, restringem os estudos sobre conteúdo e transporte de vapor d'água sobre o Brasil. Apesar das limitações mensionadas, aspectos importantes foram analisados por Rathor et al. (1987), Rao \& Marques (1984), Cavalcanti et al. (1996).

A partir de 1995, o projeto "reanálise", do National Center for Atmospheric Research/National Center for Environmental Prediction - NCAR/NCEP, proporcionou a geração de uma base de informações de variáveis meteorológicas em pontos de grade. Aplicou-se, neste processamento, o estado atual da arte em análise e previsão dentro de um rígido controle de qualidade (Kalnay et al., 1996). Esta ação teve significativa importância, para o estudo do vapor d'água na atmosfera e seu transporte visto que, no caso do Brasil, garante uma série temporal regular e pode cobrir áreas com pouca ou nenhuma medição. Em estudos mais recentes se utiliza este tipo de informações: Chen \& Pfaendtner (1993); Higgins et al. (1996); Rao et al. (1996, 1998); Cavalcanti et al. (2002).

A determinação da evapotranspiração (ET) é um processo relativamente complexo, sobretudo quando se pretende obter estimativas regionais que permitam avaliar sua distribuição sazonal e interanual. A precipitação pluviométrica $(\mathrm{P})$, por sua vez, é medida com maior facilidade e, portanto, encontra-se disponibilizada mais facilmente, mas esses parâmetros do balanço hídrico à superfície podem ser determinados como resíduo (ET-P), a partir da avaliação da divergência do fluxo de vapor d'água integrado verticalmente. A metodologia apresentada poderá ser útil para se avaliar o balanço hídrico em bacias hidrográficas a partir de prognósticos de modelos numéricos.
Nesse sentido, se objetiva no presente trabalho, estimar o resíduo (ET-P) a partir do fluxo resultante de vapor d'água sobre três áreas selecionadas das regiões Nordeste, Norte e Sudeste do Brasil e seu comportamento sazonal (intra-anual) e interanual utilizando-se dados de "reanálise".

\section{MATERIAL E MÉTODOS}

\section{Dados}

Neste estudo se utilizaram os valores mensais de umidade específica do ar (q) e componentes zonal (u) e meridional (v) do vento, para uma grade de 2,5 por $2,5^{\circ}$ de latitude e longitude, respectivamente, em seis níveis padrões (superfície, 925, 850, 700, 500, e $300 \mathrm{hPa}$ ) no período de 1958 a 1998; esses dados se referem às "reanálises" do modelo global do NCAR/NCEP.

O projeto "reanálise" do NCAR/NCEP teve início em 1995 e gerou uma base de informações de variáveis meteorológicas em pontos de grade. Neste processamento se aplicou o estado atual da arte em análise e previsão dentro de um rígido controle de qualidade, para assimilação dos dados observacionais. Diferentes plataformas de observações foram utilizadas na geração das "reanálises", dentre as quais se destacam: estações de superfície, navios, aeronaves, balões piloto, radiossondas, bóias e satélites (Kalnay et al., 1996).

Tem-se observado que as "reanálises" apresentam altos valores de umidade relativa do ar, nos níveis superiores da atmosfera, quando comparados com os valores observados mas, para o estudo em questão, isto não é relevante, dado que a concentração substancial de vapor d'água atmosférico (umidade específica) se localiza nos baixos níveis, inferior a $500 \mathrm{hPa}$.

\section{Área de estudo}

Três diferentes áreas sobre o Brasil foram definidas, de 10 por 10 graus de latitude e longitude, respectivamente, a saber: área Nordeste $\left(2,5-12,5^{\circ} \mathrm{S}\right.$ e $\left.35,0-45,0^{\circ} \mathrm{W}\right)$, área Norte $\left(0,0-10,0^{\circ} \mathrm{S}\right.$ e $\left.55,0-65,0^{\circ} \mathrm{W}\right)$ e área Sudeste $\left(15,0-25,0^{\circ} \mathrm{S}\right.$ e $\left.40,0-50,0^{\circ} \mathrm{W}\right)$. O tamanho de todas as áreas é de aproximadamente $1.232 .100 \mathrm{~km}^{2}$ (Figura 1). Em relação às grandes bacias hidrográficas, a área Nordeste cobre a maior parte da sua bacia, parte da bacia do médio São Francisco e toda a bacia do baixo São Francisco e, ainda, parte norte da bacia do Leste. Destacam-se importantes cidades contidas nesta área, tais como: Teresina (PI), Fortaleza (CE), Natal (RN), João Pessoa (PB), Recife (PE), Petrolina (PE), Maceió (AL), Aracaju (SE) e Juazeiro (BA); já a área denominada Norte, está totalmente inserida na bacia Amazônica e quase centrada na cidade de Manaus (AM). Ressalta-se que nessa área se encontra a floresta amazônica que tem importância vital para o clima do Brasil e do mundo.

Tem-se, na área Sudeste, a nascente do rio São Francisco, na denominada bacia do alto São Francisco, parte sul da bacia do Leste e parte leste da bacia do Paraná; nesta área se encontram grandes metrópoles, como: Rio de Janeiro (RJ), São Paulo (SP), Belo Horizonte (MG), Vitória (ES) e Goiânia $(\mathrm{GO})$, entre outras. 


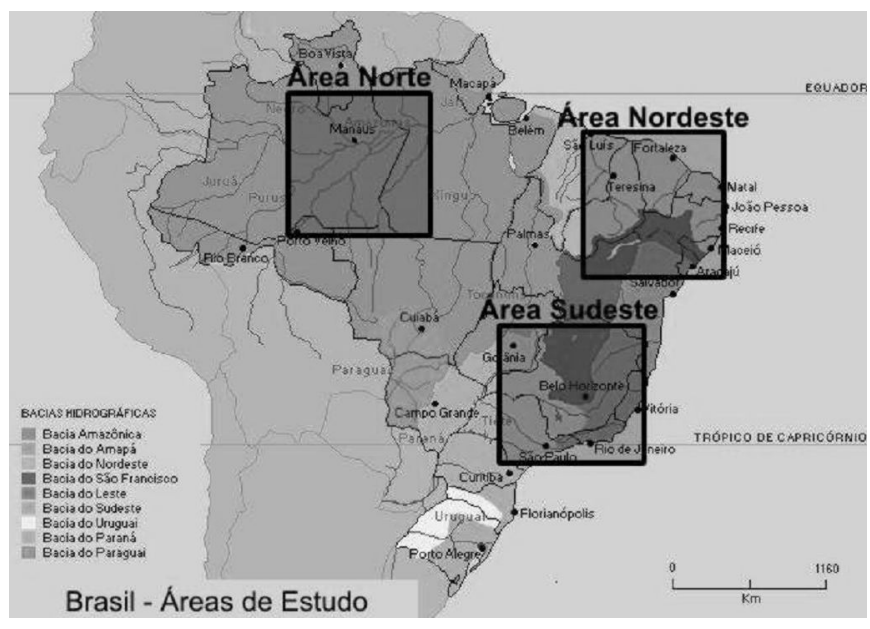

Figura 1. Localização das áreas de estudo: Nordeste $(2,5-12,5$ ㅇ e 35,0 -

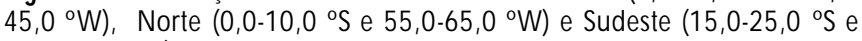
$40,0-50,0$ oW )

\section{Formulação teórica}

Para avaliação dos parâmetros do balanço d'água na atmosfera, utilizou-se a formulação apresentada por Peixoto (1973) e Peixoto \& Oort (1992). Para um ponto da atmosfera em determinado instante, o balanço de água na fase de vapor é expresso pela equação:

$$
\frac{\mathrm{Dq}}{\mathrm{Dt}}=\mathrm{S}(\mathrm{q})
$$

em que q é a umidade específica para o vapor d'água e S(q) representa uma taxa de formação (fonte) ou destruição (sumidouro) de vapor d'água por unidade de massa. O termo $S(q)=e-c$, em que "e" é a taxa de evaporação e "c" é a taxa de condensação, todos por unidade de massa. Reescrevendo a Eq. (1) e se expandindo a derivada material, ter-se-á:

$$
\frac{\partial \mathrm{q}}{\partial \mathrm{t}}+\nabla_{h} \cdot \mathrm{q} \overrightarrow{\mathrm{V}}+\frac{\partial \mathrm{q} \omega}{\partial \mathrm{p}}=\mathrm{e}-\mathrm{c}
$$

Similarmente, uma equação para o balanço na fase condensada pode ser escrita observando-se que a taxa de formação (fonte) ou destruição (sumidouro) de água na fase de condensação é $S\left(q_{c}\right)=-S(q)$ de forma que $\mathrm{S}\left(\mathrm{q}_{\mathrm{c}}\right)=-(\mathrm{e}-\mathrm{c}), \log \mathrm{o}:$

$$
\frac{\partial \mathrm{q}_{\mathrm{c}}}{\partial \mathrm{t}}+\nabla_{\mathrm{h}} \cdot \mathrm{q}_{\mathrm{c}} \overrightarrow{\mathrm{V}}+\frac{\partial \mathrm{q}_{\mathrm{c}} \omega}{\partial \mathrm{p}}=-(\mathrm{e}-\mathrm{c})
$$

em que $\vec{V}=u \vec{i}+v \vec{j}$ é o vetor velocidade do vento e $\omega$ é a velocidade vertical em coordenada isobárica.

A equação para o balanço total em determinado nível de pressão $\mathrm{P}$, é obtida somando-se as Eqs 2 e 3; portanto:

$$
\left(\frac{\partial \mathrm{q}}{\partial \mathrm{t}}+\nabla \cdot \mathrm{q} \overrightarrow{\mathrm{V}}+\frac{\partial \mathrm{q} \omega}{\partial \mathrm{p}}\right)+\left(\frac{\partial \mathrm{q}_{\mathrm{c}}}{\partial \mathrm{t}}+\nabla \cdot \mathrm{q}_{\mathrm{c}} \overrightarrow{\mathrm{V}}+\frac{\partial \mathrm{q}_{\mathrm{c}} \omega}{\partial \mathrm{p}}\right)=0
$$

A equação (4) pode ser integrada ao longo da vertical, com respeito à pressão, desde a superfície $\mathrm{P}_{\mathrm{s}}$, até um nível $\mathrm{P}_{\text {topo }}$ considerado. A equação resultante para o conteúdo total de água nas três fases (sólido, líquido e vapor) em uma coluna da atmosfera, pode ser expressa por:

$$
\frac{\partial \mathrm{W}}{\partial \mathrm{t}}+\nabla \cdot \overrightarrow{\mathrm{Q}}-\mathrm{ET}=-\frac{\partial \mathrm{W}_{c}}{\partial \mathrm{t}}-\nabla \cdot \overrightarrow{\mathrm{Q}}_{\mathrm{c}}-\mathrm{P}
$$

em que $\mathrm{W}$ e $\mathrm{W}_{\mathrm{c}}$ são os conteúdos de água nas fases de vapor e condensada, $\overrightarrow{\mathrm{Q}}$ e $\overrightarrow{\mathrm{Q}}_{\mathrm{c}}$ são os fluxos de água nas fases de vapor e condensado, ET é a evapotranspiração e P é a precipitação pluvial. O termo ET-P é denominado resíduo.

Analisando-se a equação (5) tem-se que, para escalas de tempo longas (acima de um mês) $\partial \mathrm{W} / \partial \mathrm{t}$ e $\partial \mathrm{W}_{\mathrm{c}} / \partial \mathrm{t}$ podem ser desprezados; o termo $\nabla . \vec{Q}_{c}$ é de fundamental importância em áreas pequenas e representa o transporte da água condensada existente em partículas de nuvens. Esses termos têm sido desprezados em estudos para escala de tempo de mês ou superior e áreas grandes (Rathor et al., 1987; Cavalcanti et al., 2002), ficando:

$$
\nabla \cdot \overrightarrow{\mathrm{Q}}=\mathrm{ET}-\mathrm{P}
$$

Os valores médios desses parâmetros nas áreas indicadas na Figura 1 foram avaliados conforme volume de controle esquematizado pela Figura 2 e calculados pela expressão:

$$
\frac{1}{\mathrm{~A}} \iint_{\mathrm{A}} \nabla \cdot \overrightarrow{\mathrm{Q}} \mathrm{dA}=\langle\mathrm{ET}-\mathrm{P}\rangle
$$

que, após a aplicação do teorema de Gauss, fica:

$$
\frac{1}{A} \oint_{1} \overrightarrow{\mathrm{Q}} \cdot \overrightarrow{\mathrm{n}} \mathrm{dl}=\langle\mathrm{ET}-\mathrm{P}\rangle
$$

em que $\overrightarrow{\mathrm{n}}$ é o vetor unitário normal à face lateral do volume, dl é um elemento infinitesimal da face lateral, $\overrightarrow{\mathrm{Q}}=\frac{1}{\mathrm{~g}} \int_{300}^{\mathrm{P}_{\mathrm{wp}} \mathrm{q}} \mathrm{q} \overrightarrow{\mathrm{V}} \mathrm{dp}$ é o fluxo de vapor d'água integrado vertical-

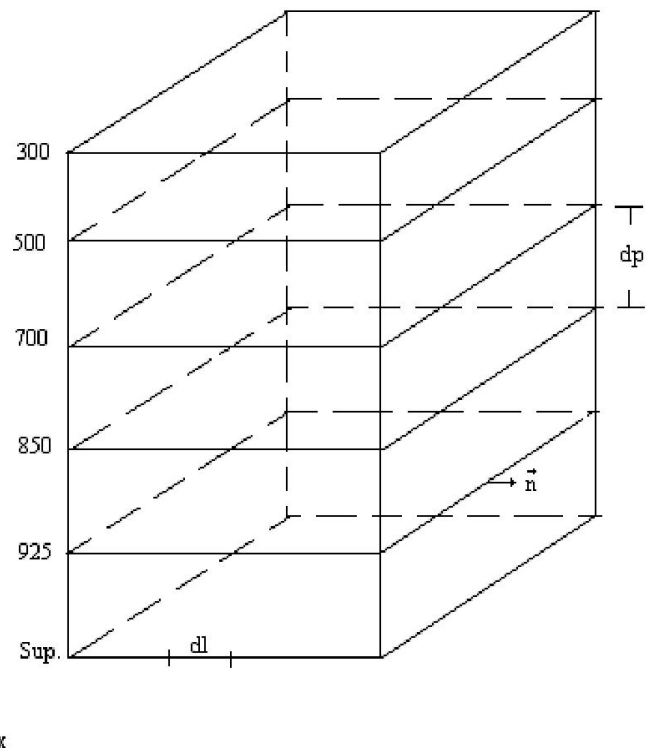

Figura 2. Esquema gráfico para o volume de controle 
mente e $\left\langle(\right.$ ) $\rangle=\frac{1}{\mathrm{~A}} \iint_{\mathrm{A}}(\mathrm{)}) \mathrm{dA}$ é a expressão da média de ( ) na área A. O termo do lado esquerdo da Eq. (8) representa o Fluxo Resultante (FR).

\section{RESULTADOS E DISCUSSÃO}

Os valores médios mensais de precipitação pluvial, evapotranspiração e fluxo resultante de vapor d'água, para cada área, no período de 1958 a 1998, representam uma composição dos sub-regimes de cada região com períodos chuvoso e seco, ao longo do ano, caracterizado pela variação sazonal. A própria resolução espacial da "reanálise" contribui para uma macro caracterização desses parâmetros; na área Norte, entretanto, esta variação é menos acentuada quando comparada às áreas Nordeste e Sudeste; conseqüentemente, apresenta um comportamento mais regular durante todo o ano (Figura 3); mesmo assim, ocorrem diferentes sub-regimes de precipitação na área. Referida figura foi construída de modo que o período chuvoso ficasse relativamente centralizado no eixo das abscissas.

Observa-se, na Figura 3A, que na área Nordeste a precipitação pluvial máxima se deu no mês central do trimestre chuvoso fevereiro-março-abril, com valor médio diário de 8,7 $\mathrm{mm} \mathrm{dia}{ }^{-1}$, totalizando $270 \mathrm{~mm}$ mês $^{-1}$; já no trimestre menos chuvoso do ano, agosto-setembro-outubro, o mês de setembro se apresenta com média diária de $1,8 \mathrm{~mm} \mathrm{dia}^{-1}$, que totaliza $54 \mathrm{~mm}$ mês ${ }^{-1}$, entretanto a maior taxa de evapotranspiração, no mês de fevereiro, apresentou média de 4,2 $\mathrm{mm}$ $\mathrm{dia}^{-1}$, cujos fluxos residual (ET-P) e resultante (FR) foram máximos, com valores absolutos de 4,6 e 3,8 $\mathrm{mm} \mathrm{dia}^{-1}$, respectivamente. Comparando-se ET-P e FR para os demais meses, verifica-se a existência de relação linear confirmando, assim, a formulação da Eq. (8); o sinal negativo para o FR indica que a precipitação pluvial superou a evapotranspiração e o sinal positivo mostra o contrário.

Na área Norte (Figura 3B) a precipitação pluvial é da ordem de $8 \mathrm{~mm} \mathrm{dia}^{-1}$ (240 $\mathrm{mm}$ mês $\left.{ }^{-1}\right)$ para os meses de outubro a abril; este valor se aproxima do observado na área Nordeste durante o trimestre mais chuvoso. Os meses restantes (maio a setembro), ainda apresentam precipitações consideráveis de 5 a $7 \mathrm{~mm} \mathrm{dia}{ }^{-1}\left(150\right.$ a $\left.210 \mathrm{~mm}^{\text {mês }}{ }^{-1}\right)$. A evapotranspiração se mantém durante todo o ano, entre $4 \mathrm{e}$ $5 \mathrm{~mm} \mathrm{dia}{ }^{-1}$ (120 e $\left.150 \mathrm{~mm} \mathrm{mês}^{-1}\right)$. A relação entre o resíduo ET-P e o FR aparenta-se bem melhor que o observado na área Nordeste; não se observa déficit hídrico para esta área.

Na Figura 3C, área Sudeste, o mês de janeiro apresentou o maior valor de precipitação $\left(7 \mathrm{~mm} \mathrm{dia}{ }^{-1}, 217 \mathrm{~mm}\right.$ mês $\left.{ }^{-1}\right)$ em relação aos demais meses do ano. Valores bem próximos ao de janeiro têm os meses de dezembro a fevereiro que, juntos, formam o trimestre mais chuvoso do ano. A evapotranspiração apresentou a maior variação sazonal comparada às demais áreas, variando entre 2 e 4,5 mm dia ${ }^{-1}$. Verifica-se deficiência hídrica de maio a agosto, evidenciada pelo sinal positivo do FR. A relação entre o resíduo e o fluxo resultante também se mostra boa.

Apresentam-se, nas Figuras 4, 5 e 6, as variações interanuais das variações sazonais da precipitação pluvial, eva-
A.

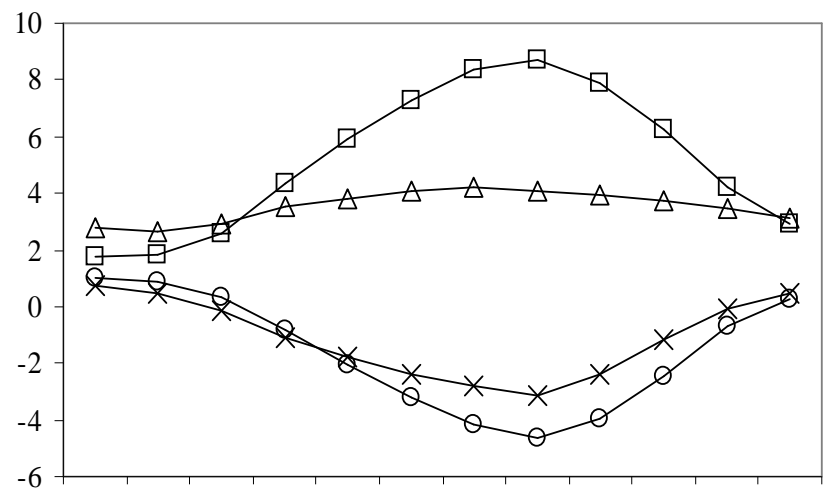

B.

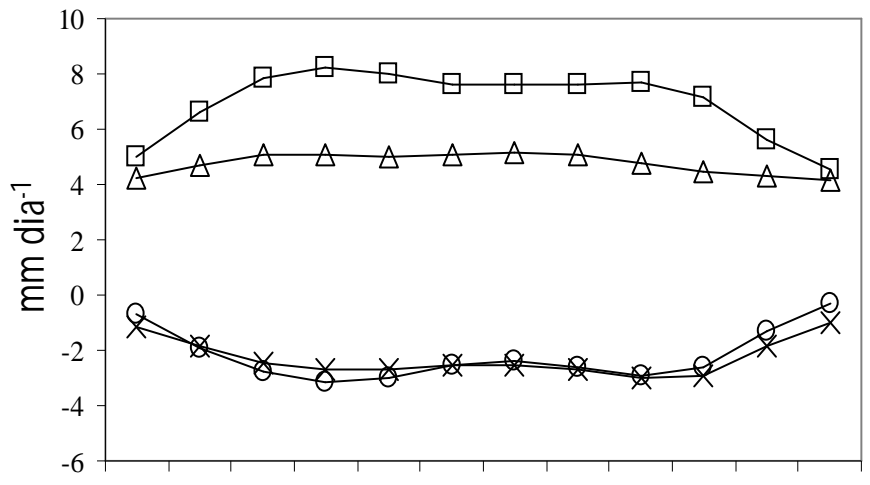

C.

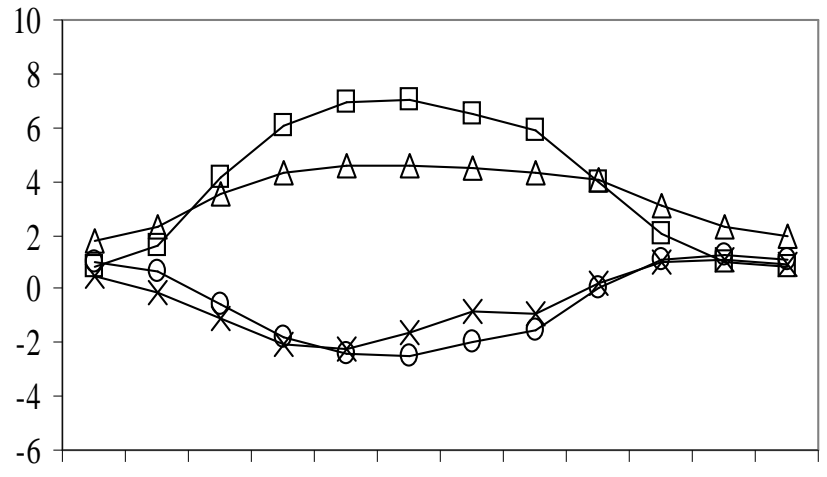

Ago Set Out Nov Dez Jan Fev Mar Abr Mai Jun Jul Mês

七-P $\triangle$ ET -0 - ET-P $\nsucc$ FR

Figura 3. Média mensal (1958-1998) da precipitação pluvial (P), evapotranspiração (ET), resíduo (ET-P) e fluxo resultante (FR) para: A) área Nordeste, B) área Norte e C) área Sudeste

potranspiração, resíduo ET-P e fluxo de vapor d'água resultantes, para as áreas Nordeste, Norte e Sudeste, respectivamente.

Em relação à precipitação pluvial na área Nordeste (Figura 4A) vê-se que os trimestres mais chuvosos do ano (fevereiro-abril) e o mais seco (agosto-outubro) estão presentes em todos os anos do período analisado (1958 a 1998). A evapotranspiração (Figura 4B) segue o mesmo padrão de distribuição, evapotranspirando mais quando há maior disponibilidade de água no solo e evapotranspirando menos no 
A.

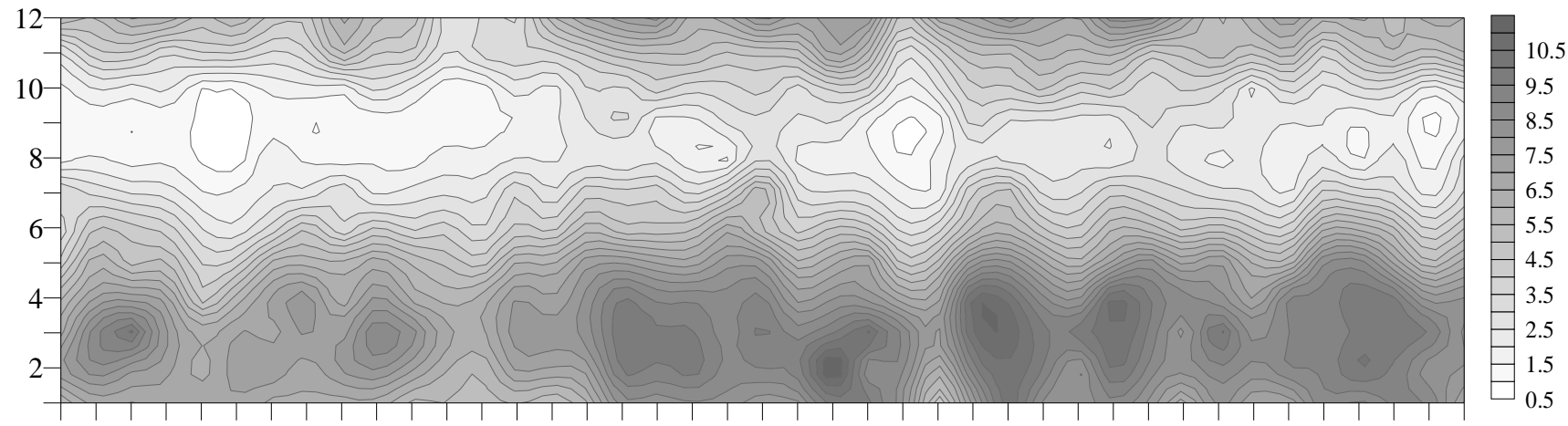

B.

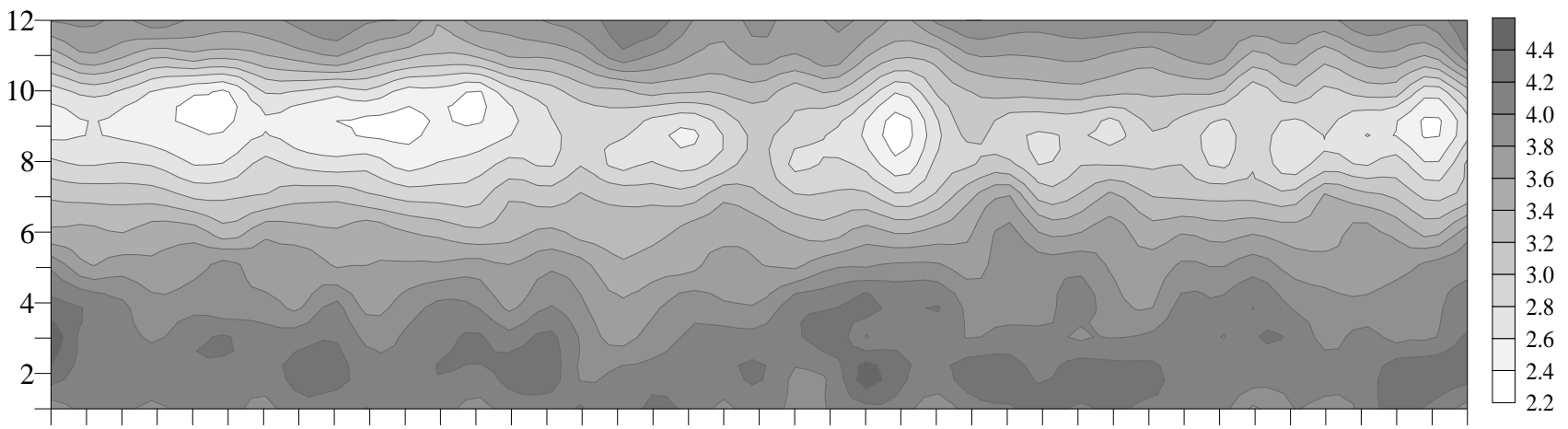

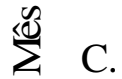

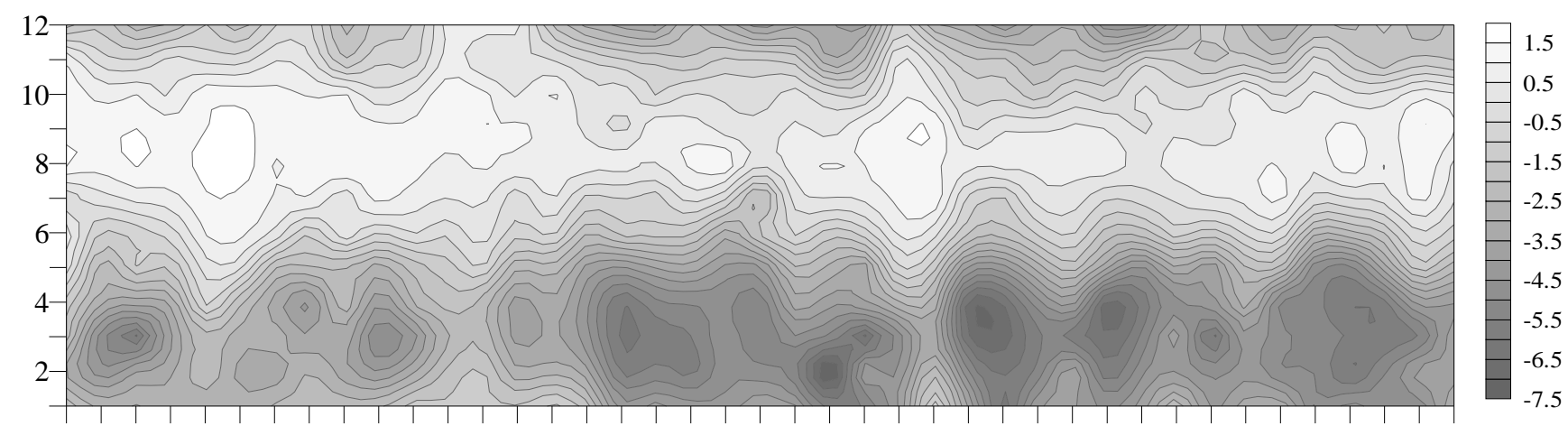

D.

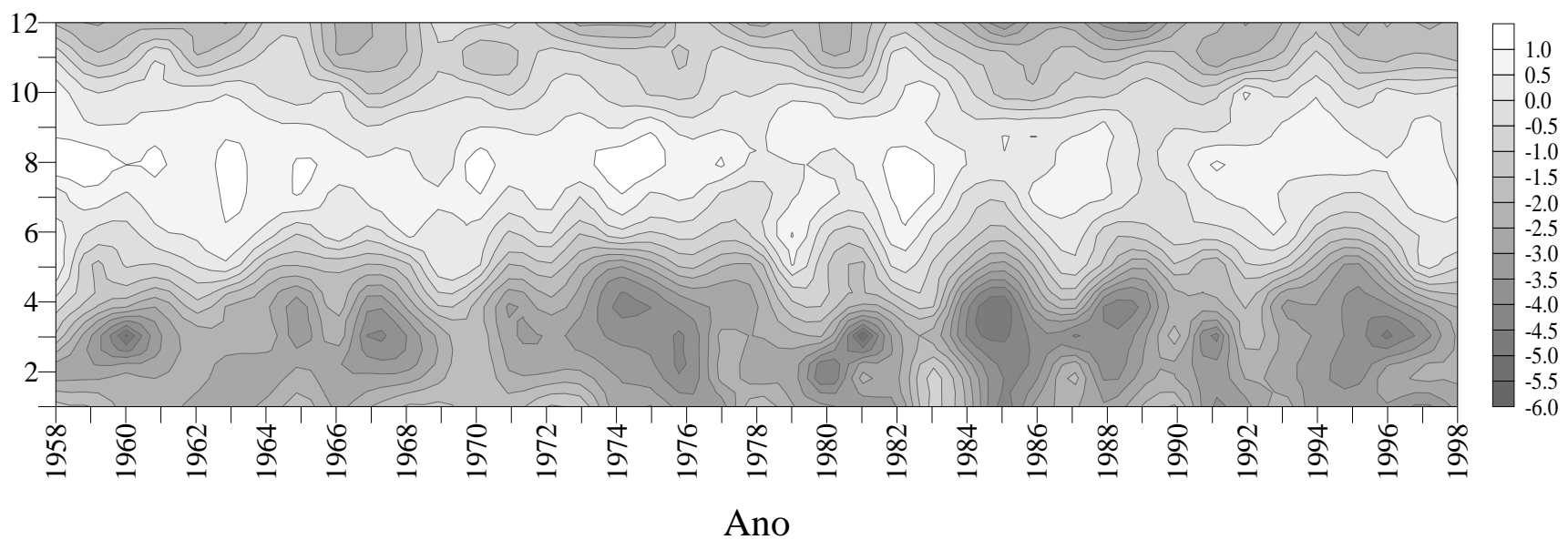

Figura 4. Distribuição anual (1958-1998) dos valores mensais de: A) Precipitação (P); B) Evapotranspiração (ET); C) Resíduo (ET-P) e D) Fluxo Resultante (FR) para a área Nordeste ( $m m$ dia $\left.^{-1}\right)$ 
A.

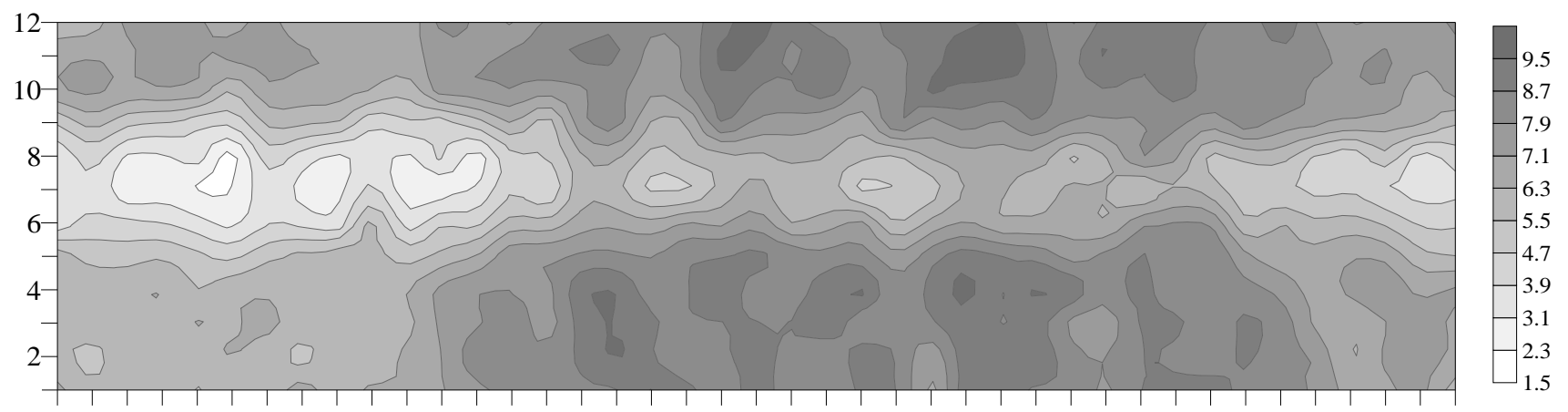

B.

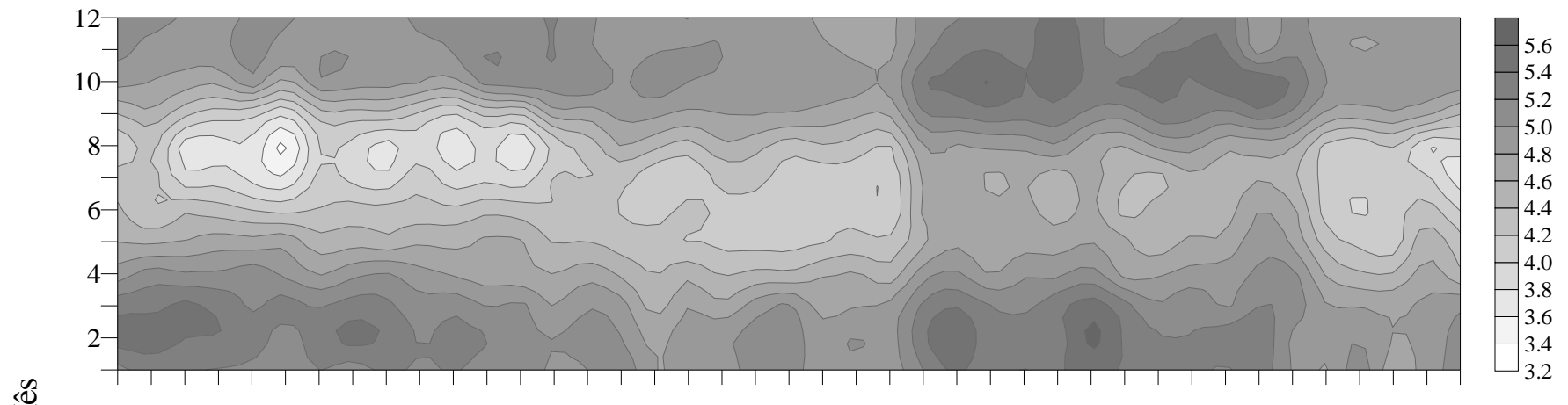

C.

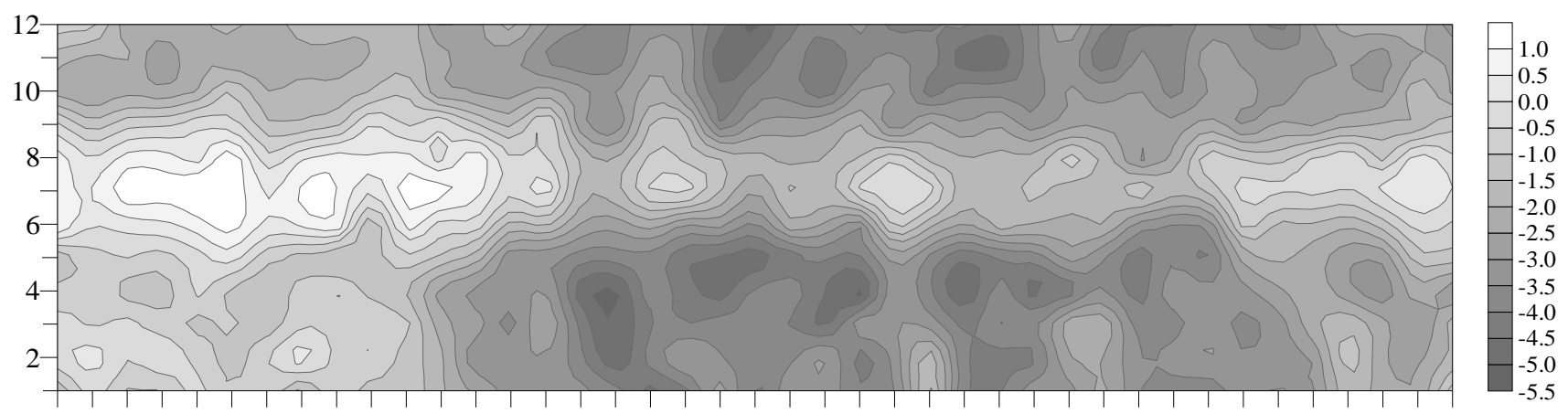

D.

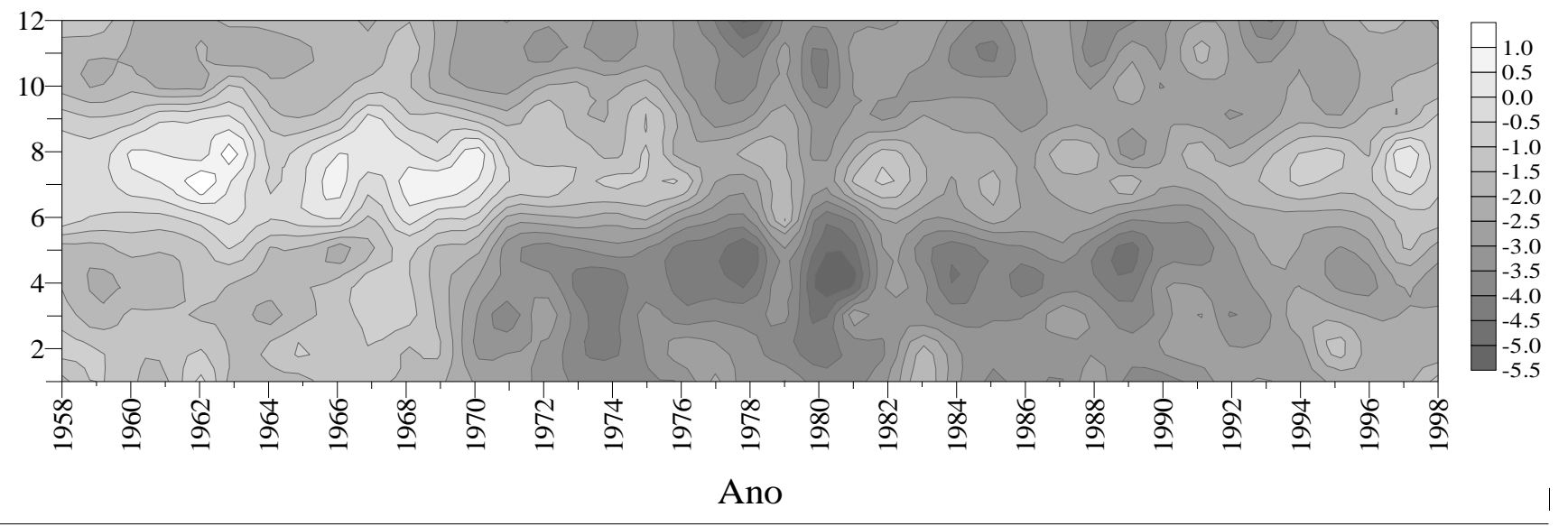

Figura 5. Distribuição anual (1958-1998) dos valores mensais de: A) Precipitação (P); B) Evapotranspiração (ET); C) Resíduo (ET-P) e D) Fluxo Resultante (FR) para a área Norte (mm dia-1) 
A.

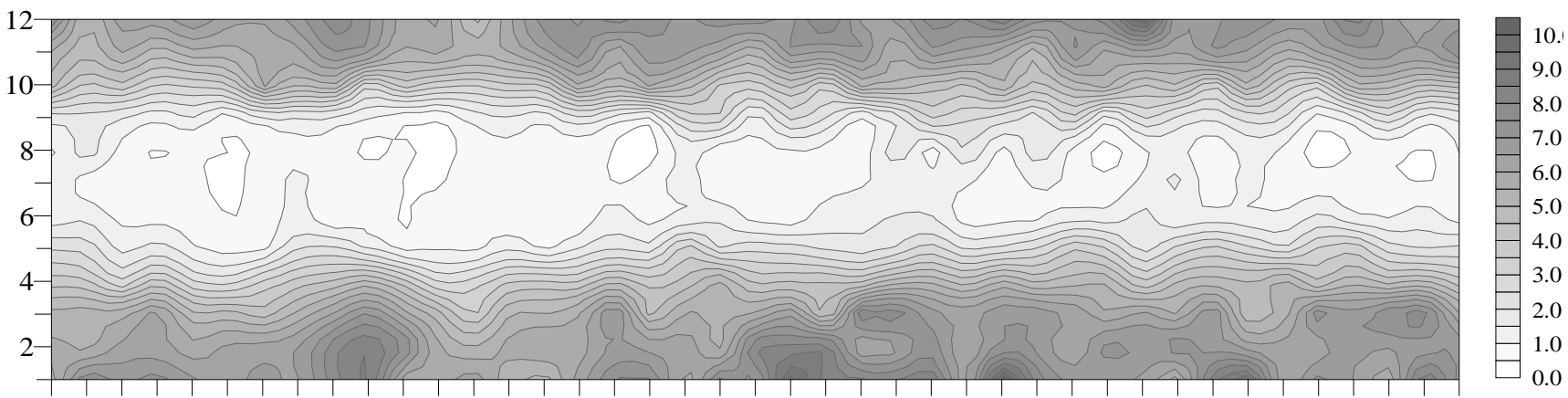

B.

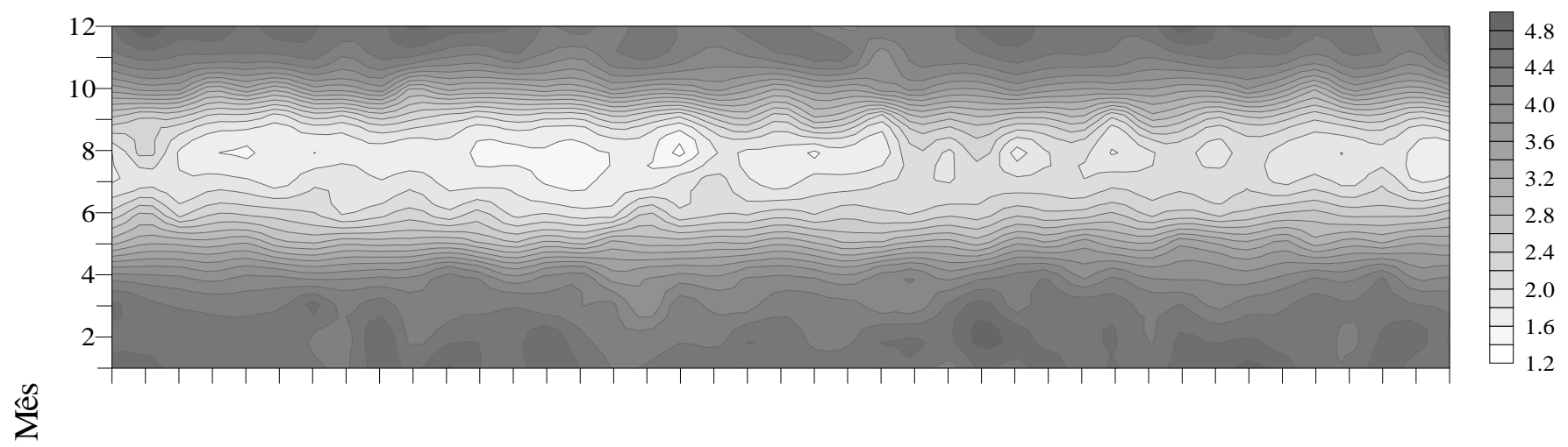

C.

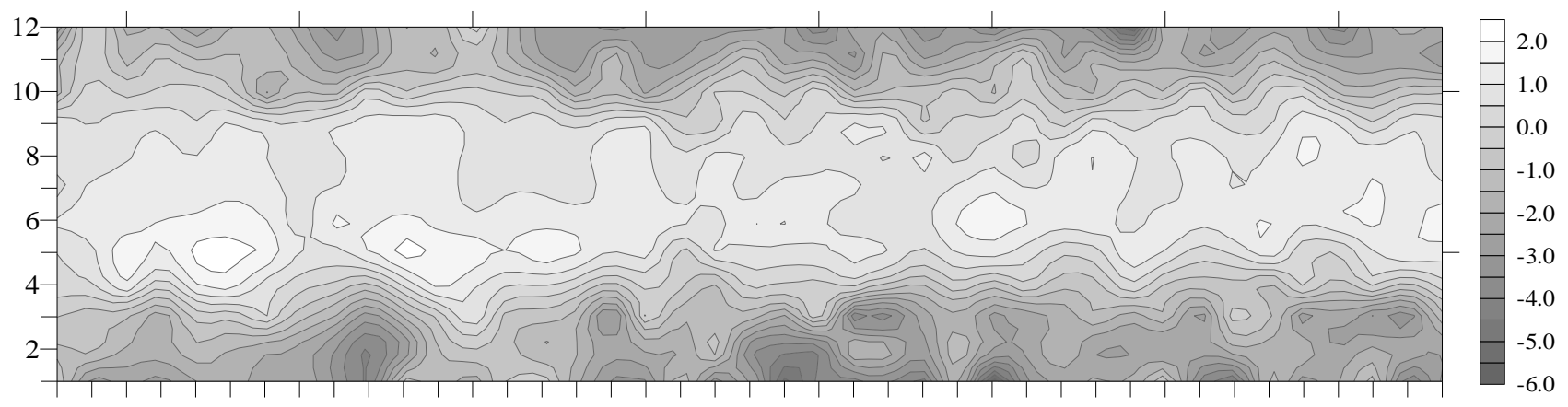

D.

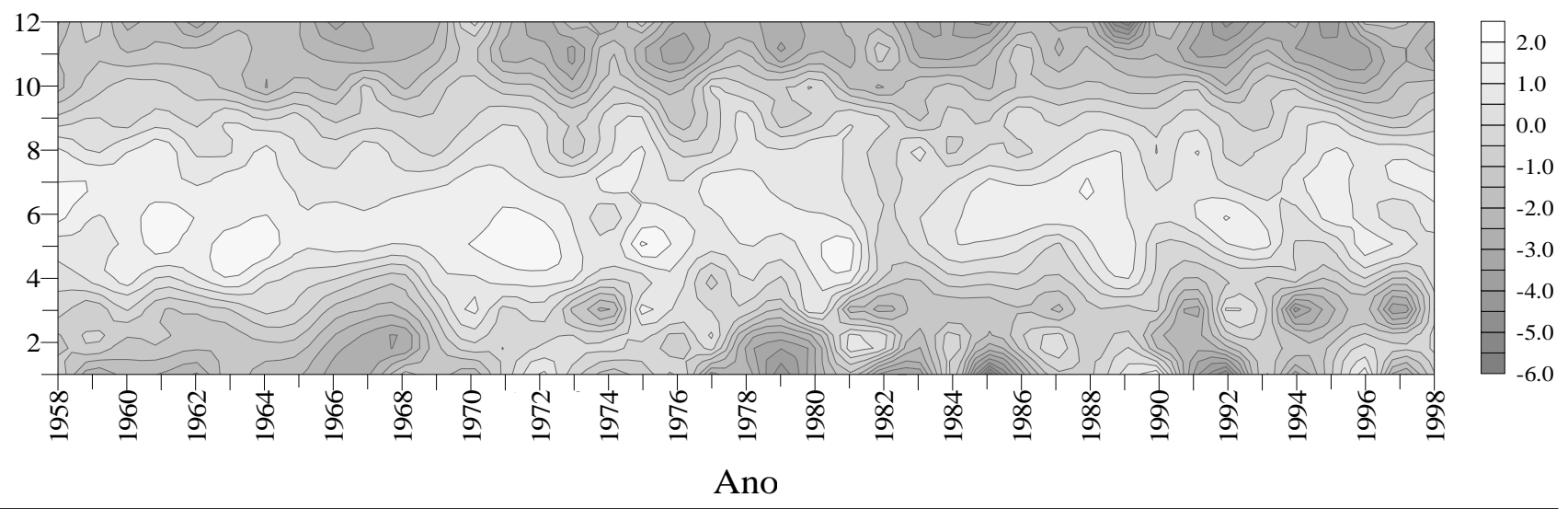

Figura 6. Distribuição anual (1958-1998) dos valores mensais de: A) Precipitação (P); B) Evapotranspiração (ET); C) Resíduo (ET-P) e D) Fluxo Resultante (FR) para a área Sudeste $\left(\mathrm{mm} \mathrm{dia}^{-1}\right)$ 
período mais seco do ano. Comparando-se as Figuras $4 \mathrm{C}$ e $\mathrm{D}$, conclui-se que, além de apresentarem características sazonais descritas anteriormente, apresentam boa semelhança em cada ano, dado que associam altos (baixos) valores de fluxos de vapor d'água resultante a altos (baixos) valores de resíduos (ET-P) e os valores negativos indicam que a precipitação excede a evapotranspiração (entra mais vapor d'água no volume de controle do que sai) e os valores positivos mostram o contrário.

A variação nos diversos anos estudados indica a ocorrência de anos de intensa precipitação e anos de moderada ou fraca precipitação, caracterizando-os como anos chuvosos, normais e secos, respectivamente, além de boa relação do resíduo com o fluxo resultante de vapor d'água e que, nos anos chuvosos, o fluxo de vapor d'água resultante é negativamente mais intenso, indicando forte sumidouro de vapor d'água na atmosfera, ou seja, maior quantidade de vapor d'água se condensando e, conseqüentemente, precipitando; este resultado é comprovado pelo resíduo (Figura 4C) em que fortes núcleos de precipitação excedem a evapotranspiração.

$\mathrm{Na}$ área Norte a ocorrência de precipitação (Figura 5A) é bem distribuída ao longo do ano, em um número maior de meses: de outubro a abril; esta característica climática é verificada praticamente em todos os anos considerados chuvosos ou menos chuvosos, já que ano seco não é uma característica marcante nessa área.

A evapotranspiração (Figura 5B) apresenta uma variação anual e interanual mais uniforme, em comparação com as outras áreas, devido à disponibilidade de água no solo durante todo o ano visto que, em média, não há deficiência hídrica. O período de menor evapotranspiração parece ser regulado pela menor disponibilidade de energia solar em junho, julho e agosto.

A semelhança entre as Figuras 5C e D, indica a relação entre o resíduo ET-P e o fluxo resultante de vapor d'água, de forma idêntica ao que ocorre para a área Nordeste. Observa-se, entre os anos analisados, que a freqüência de meses em que a evapotranspiração é superior à precipitação, é bem reduzida em comparação com outras áreas estudadas e, quando ocorre, compreende apenas cerca de dois a três meses.

A Figura 6 apresenta a variação interanual para o período de 1958 a 1998, da precipitação (A), evapotranspiração (B), diferença ET-P (C) e do fluxo resultante (D) de vapor d'água atmosférico para a região sudeste. A análise evidencia que o período chuvoso na região se situa entre os meses de outubro a abril e o período seco, de maio a setembro. Em alguns anos, tal como em 1992 e 1993, há um período de estiagem nos meses de março e abril, em que o período seco apresenta valores mínimos de ET (Figura 6B). Através da Figura 6C observa-se, pelo comportamento da diferença ET$\mathrm{P}$, que para o período estudado os maiores valores positivos ocorrem durante boa parte do ano (abril a outubro), configurando uma evapotranspiração maior que a precipitação pluvial; somente no período chuvoso, nos meses de novembro a março, essa diferença fica negativa com precipitação sendo maior que a evapotranspiração. Através da Figura 6D verifica-se que o fluxo de vapor d'água atmosférico resultante é positivo na maior parte do ano, indicando que a região é fonte de vapor exportando mais do que importando.

A Figura 7 expressa a relação entre os fluxos residual ET$\mathrm{P}$ e de vapor d'água resultante FR, para as três áreas em estudo, observando-se coeficientes de determinação de 0,86 , 0,84 e 0,74 para as áreas Nordeste, Norte e Sudeste, respectivamente; esses coeficientes são considerados bons. Os resultado aponta para a possibilidade de se utilizar esta metodologia em modelos numéricos da atmosfera para prever o resíduo em uma área, por exemplo: uma bacia hidrográfica. Os valores de precipitação e evapotranspiração fornecidos diretamente pelos modelos atmosféricos são parametrizados e, portanto, ainda não alcançam, na sua maioria, a precisão desejada. Os campos de umidade específica do ar e a velocidade do vento são obtidos de equações prognósticas baseadas em princípios físicos, o que garante uma boa previsão e, conseqüentemente, também boa estimativa do resíduo ET-P.

\section{A.}

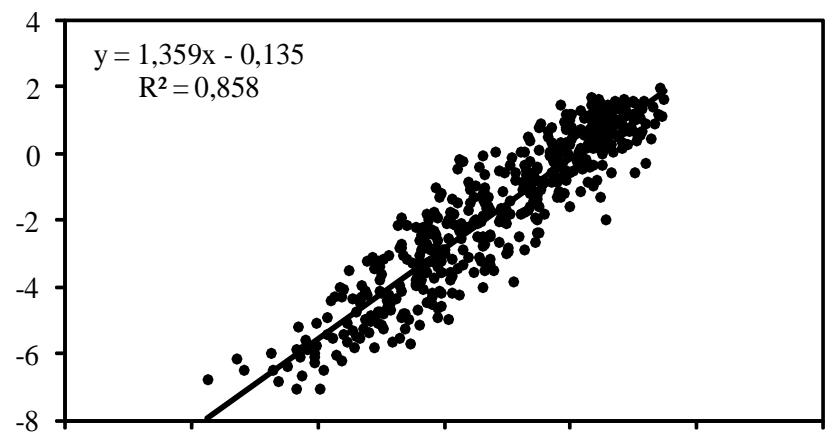

B.

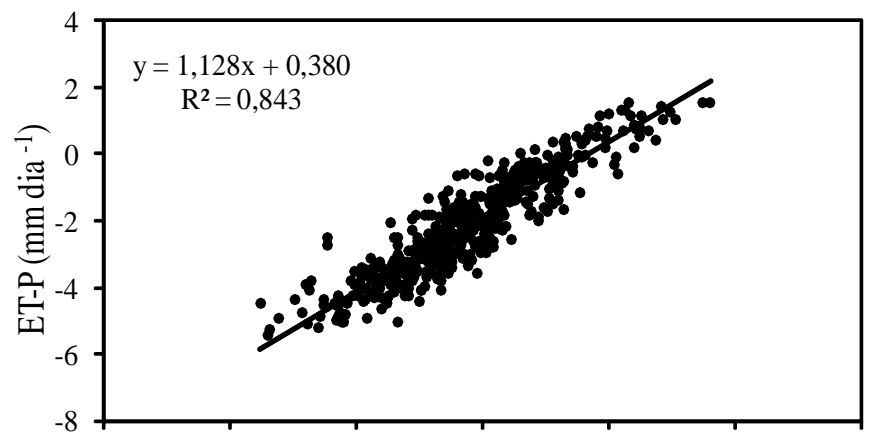

C.

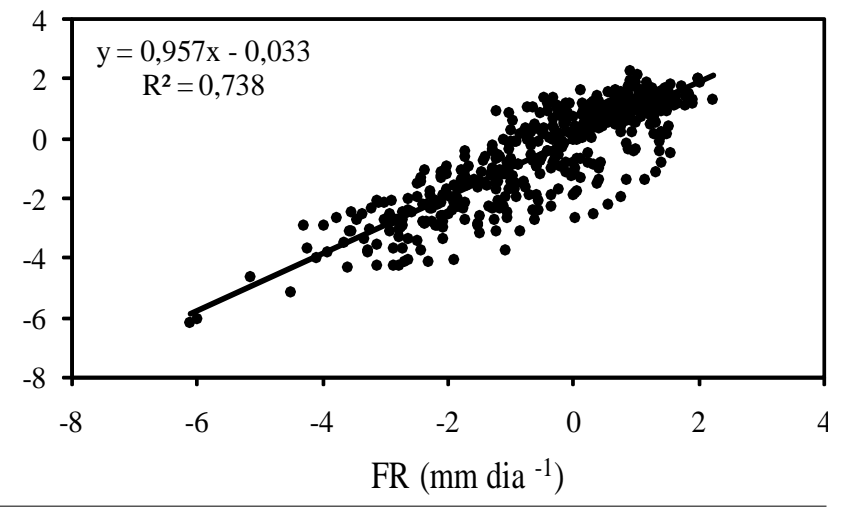

Figura 7. Relação entre o Resíduo (ET-P) e o Fluxo Resultante (FR) para: A) área Nordeste, B) área N orte e C) área Sudeste 


\section{CONCLUSÕES}

1. Nos diferentes períodos chuvosos das três regiões, a evapotranspiração é maior, especialmente no início do período, assim como o resíduo ET-P.

2. Com exceção da área norte, há um déficit hídrico no período seco das outras regiões, caracterizado por valores positivos de ET-P.

3. Comparativamente, há maior variação sazonal da evapotranspiração na região sudeste que nas outras duas regiões.

4. As áreas nas quais ocorre déficit hídrico à superfície, podem ser consideradas fontes de vapor para a atmosfera, pois apresentam fluxo resultante positivo, compatível com divergência do fluxo sobre a área.

5. As regiões norte e nordeste apresentam valores semelhantes de precipitação na época chuvosa, porém na região norte a precipitação se mantém durante todo o ano.

\section{LITERATURA CITADA}

Benton, G. S.; Estoque, M. A. Water-vapour transfer over the North American Continent. Journal of Meteorology, v.11, n.6, p.462-477, 1954.

Brubaker, L. K.; Entekhabi, D.; Eagleson, P. S. Estimation of continental precipitation recycling. Journal of Climate, v.6, n.6, p.1077-1089, 1993.

Cavalcanti, E. P.; Ceballos, J. C.; Valadão, C. E. de A. Vapor d'água sobre Campina Grande, Barbalha e Fortaleza durante o experimento "EMAS". In: Congresso Brasileiro de Meteorologia, 9, 1996, Campos do Jordão. Anais.... Campos do Jordão: SBMET., 1996. p.850-854.

Cavalcanti, E. P.; Gandu, A. W.; Azevedo, P. V. Transporte e balanço de vapor d'água atmosférico sobre o Nordeste do Brasil. Revista Brasileira de Meteorologia, v.17, n.2, p. 207-217, 2002.

Chen, T. C.; Pfaendtner, J. On the atmospheric branch of the hydrological cycle. Journal of Climate, v.6, n.1, p.161-167, 1993.

Hastenrath, S. L. The flux of atmospheric water vapor over Caribbean Sea and Gulf of Mexico. Journal of Applied Meteorology, v.5,n.6p.778-788, 1966.
Higgins, R. W.; Mo, K. C.; Schubert, S. D. The moisture budget of the central United States in springs as evaluated in the NCEP/NCAR and NASA/DAO reanalyses. Monthly Weather Review, v.124, n.5, p.939-963, 1996.

Howarth, D. A. Seasonal variations in the vertically integrated water vapor transport fields over the Southern Hemisphere. Monthly Weather Review, v.111, n.6, p.1259-1272, 1983.

Kalnay, E.; Kanamitsu, M.; Kistler, R.; Collins, W.; Deaven, D.; Gandin L.; Iredell, M.; Saha, S.; White, G; Woollen, J.; Zhu, Y.; Leetmaa, A.; Reynolds, B.; Chelliah, M.; Ebisuzaki, W.; Higgins, W.; Janowiak, J.; Mo, K.C.; Ropelewski, C.; Wang, J.; Roy, J. R.; Joseph, D. The NCEP/NCAR 40-year reanalysis project. Bulletin of the American Meteorological Society, v.77, n.3, p.437-471, 1996.

Peixoto, J. P. O campo da divergência do transporte de vapor de água na atmosfera. Revista da Faculdade de Ciências de Lisboa, v.7, p.25-56, 1959.

Peixoto, J. P. Atmospheric vapor flux computations for hydrological purposes. World Meteorological Organization, Geneve: WMO/IHD, 1973, n.357, 83p. Report n.20

Peixoto, J. P.; Oort, A. H. Physics of climate. New York: American Institute of Physics, 1992. 520p.

Rao, V. B.; Cavalcanti, I. F. A.; Hada, K. Annual variation of rainfall over Brazil and water vapor characteristics over South America. Journal of Geophyssical Research, v.101, n.D21 p.26539-26551, 1996.

Rao, V. B.; Chapa, S. R.; Cavalcanti, I. F. A. Moisture budget in the tropics and the Walker Circulation. Journal of Geophyssical Research, v.103, p.13713-13728, 1998.

Rao, V. B.; Marques, V. da S. Water vapor characteristics over Northeast Brazil during two contrasting years. Journal of Climate and Applied Meteorology, v.23, n.3, p.440-444, 1984.

Rasmusson, E. M. Atmospheric water vapor transport and the water balance of North America: Part I. Characteristics of the water vapor flux field. Monthly Weather Review, v.95, n.7. p.403-426, 1967.

Rasmusson, E. M. A study of hydrology of eastern North America using atmospheric vapor flux data. Monthly Weather Review, v.99, n.2, p.119-135, 1971.

Rathor, H. S.; Araújo, M. A. A.; Mello, C. C. Atmospheric water vapour flux and its divergence over the drought prone region of tropical Northeast Brazil. Revista Brasileira de Geofísica, v.3, p.107-113, 1987. 